Review

\title{
Comparing the Efficacy of OnabotulinumtoxinA, Sacral Neuromodulation, and Peripheral Tibial Nerve Stimulation as Third Line Treatment for the Management of Overactive Bladder Symptoms in Adults: Systematic Review and Network Meta-Analysis
}

\author{
Chi-Wen Lo ${ }^{1,2,3}$, Mei-Yi Wu ${ }^{4} \oplus$, Stephen Shei-Dei Yang ${ }^{2,3}$, Fu-Shan Jaw ${ }^{1}$ and \\ Shang-Jen Chang $2,3, *$ \\ 1 Institute of Biomedical Engineering, National Taiwan University, Taipei 10617, Taiwan; \\ chiwenlo0216@gmail.com (C.-W.L.); jaw@ntu.edu.tw (F.-S.J.) \\ 2 Division of Urology, Department of Surgery, Taipei Tzu Chi Hospital, The Buddhist Tzu Chi Medical \\ Foundation, New Taipei 23142, Taiwan; urolyang@tzuchi.com.tw \\ 3 School of Medicine, Buddhist Tzu Chi University, Hualien 97071, Taiwan \\ 4 Department of Nephrology, Taipei Medical University-Shuang Ho Hospital, Taipei 23561, Taiwan; \\ e220121@gmail.com \\ * Correspondence: krissygnet@gmail.com; Tel.: +886-2-66289779
}

Received: 11 December 2019; Accepted: 5 February 2020; Published: 18 February 2020

\begin{abstract}
The American Urological Association guidelines for the management of non-neurogenic overactive bladder $(\mathrm{OAB})$ recommend the use of Onabotulinumtoxin $\mathrm{A}$, sacral neuromodulation (SNM), and peripheral tibial nerve stimulation (PTNS) as third line treatment options with no treatment hierarchy. The current study used network meta-analysis to compare the efficacy of these three modalities for managing adult $\mathrm{OAB}$ syndrome. We performed systematic literature searches of several databases from January 1995 to September 2019 with language restricted to English. All randomized control trials that compared any dose of OnabotulinumtoxinA, SNM, and PTNS with each other or a placebo for the management of adult $\mathrm{OAB}$ were included in the study. Overall, 17 randomized control trials, with a follow up of 3-6 months in the predominance of trials (range 1.5-24 months), were included for analysis. For each trial outcome, the results were reported as an average number of episodes of the outcome at baseline. Compared with the placebo, all three treatments were more efficacious for the selected outcome parameters. OnabotulinumtoxinA resulted in a higher number of complications, including urinary tract infection and urine retention. Compared with OnabotulinumtoxinA and PTNS, SNM resulted in the greatest reduction in urinary incontinence episodes and voiding frequency. However, comparison of their long-term efficacy was lacking. Further studies on the long-term effectiveness of the three treatment options, with standardized questionnaires and parameters are warranted.
\end{abstract}

Keywords: network meta-analysis; OnabotulinumtoxinA; overactive bladder; peripheral tibial nerve stimulation; sacral neuromodulation

Key Contribution: Updated systematic review to compare the efficacy of three existing third line treatments for the management of adult overactive bladder using network meta-analysis. 


\section{Introduction}

Overactive bladder (OAB) syndrome is defined as "the presence of urinary urgency, usually accompanied by frequency and nocturia, with or without urgency urinary incontinence, in the absence of urinary tract infection (UTI) or other obvious pathology" [1]. The prevalence of OAB syndrome increases with age and there is no significant gender difference [2]. Non-neurogenic OAB impairs the patient's quality of life (QoL) and behavioral therapy is recommended as the first line treatment. If behavioral therapy fails, oral medications, including antimuscarinics and $\beta 3$ agonists, are recommended as the second line therapy [3]. When there is inadequate symptom control or intolerable side effects due to second line management, the American Urological Association (AUA) guidelines recommend either OnabotulinumtoxinA, sacral neuromodulation (SNM), or peripheral tibial nerve stimulation (PTNS) as third line therapy options for OAB symptoms. Third line therapy is undertaken if the patient desires further treatment and is willing to engage in treatment, and/or further treatment is determined by clinicians to be in the patient's best interests. At present, the decision on which third line therapy to perform is based on the clinicians' and patient's preference, and there is not an evidence-based hierarchy available for guidance [3].

There have been several previously published randomized control studies, which compared pairwise treatments with a placebo [4,5]. However, there has not been a direct comparison of the three available treatments, and there has also been a lack of efficiency and safety comparisons between the three treatment options. When multiple treatment modalities are considered, a network meta-analysis could help compare their efficacies. Therefore, we conducted a systemic review to compare the efficacy of OnabotulinumtoxinA, SNM, and PTNS for the treatment of OAB symptoms, using a network meta-analysis.

\section{Results}

\subsection{Included Studies}

A Preferred reporting items for systematic reviews and meta-analyses (PRISMA) flow diagram flowchart summarizing the literature search is shown in Figure 1 [6]. The initial search identified 1940 and 5722 potential studies from PubMed and EMBASE, respectively. After the removal of duplicates the total number of articles was 7662. After screening, a total of 5738 articles were excluded based on their title and/or abstract, while another 185 articles were removed after a full-text assessment. A total of 20 articles met the qualitative inclusion criteria, while 17 trials, including 3038 participants, met the criteria for systematic review and network meta-analysis. 


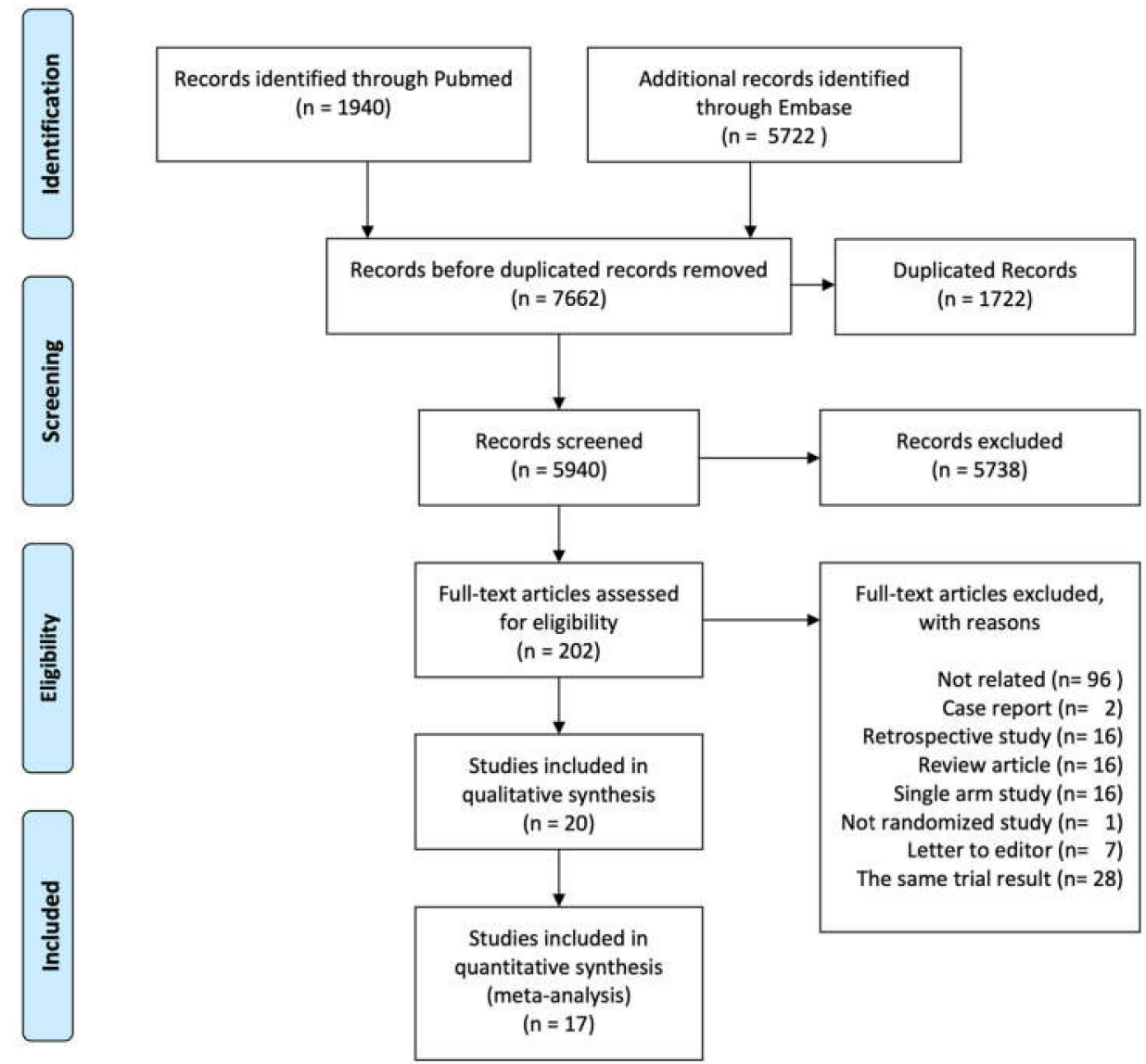

Figure 1. Preferred reporting items for systematic reviews and meta-analyses (PRISMA) flow diagram of the study selection process for network meta-analysis. The figure was generated using the PRISMA 2009 Flow Diagram.

\subsection{Study and Participant Characteristics}

The number of patients, the study design, and the inclusion and exclusion criteria for each of the included studies are listed in Table 1. As the three investigated treatment modalities are used for third line $\mathrm{OAB}$ syndromes management, most of the included patients were refractory or intolerant to the first and second line treatments. 
Table 1. Characteristics of the included randomized controlled trials.

\begin{tabular}{|c|c|c|c|c|c|c|c|}
\hline Author, Year [ref.] & $\begin{array}{c}\text { Trial } \\
\text { Registration }\end{array}$ & Study Design & Participants & Exclusion Criteria & Group Sample & $\begin{array}{l}\text { Follow-up } \\
\text { (month) }\end{array}$ & Outcomes \\
\hline \multicolumn{8}{|c|}{ OnabotulinumtoxinA vs. placebo } \\
\hline 2007 Sahai [7] & $\begin{array}{l}\text { ISRCTN } \\
16995641\end{array}$ & $\begin{array}{l}\text { Randomized, } \\
\text { Double blinded }\end{array}$ & $\begin{array}{l}\text { OAB symptoms }>6 \\
\text { months, refractory or } \\
\text { intolerant to } \\
\text { medication }\end{array}$ & $\begin{array}{l}\text { Neurological disease, } \mathrm{BOO}, \\
\text { anticoagulant therapy, } \\
\text { pregnancy, IC, indwelling } \\
\text { catheter, PVR }>200 \mathrm{~mL}, \\
\text { previous bladder surgery, UC, } \\
\text { UTI, neuromuscular } \\
\text { transmission disease }\end{array}$ & $\begin{array}{l}\text { Cystoscopy injection } \\
\text { OnabotulinumtoxinA } \\
200 \mathrm{U}(n=16) \text { vs. } \\
\text { Cystoscopy Injection } \\
\text { with Placebo }(n=18)\end{array}$ & 6 & $\begin{array}{l}\text { Change in MMC, } \\
\text { Urgency, UUI, urinary } \\
\text { frequency/day, IIQ-7, } \\
\text { UDI-6, MBC, PVR, } \\
\text { UTI, CIC }\end{array}$ \\
\hline 2009 Flynn [8] & N/A & $\begin{array}{l}\text { Randomized, } \\
\text { Double blinded }\end{array}$ & $\begin{array}{l}\text { OAB symptoms with } \\
\text { UUI, refractory to } \\
\text { medication, multiple } \\
\text { daily incontinence and } \\
\text { pad weight/day > } \\
100 \mathrm{gm}\end{array}$ & $\begin{array}{l}\text { Neurological condition, fecal } \\
\text { incontinence or absent } \\
\text { detrusor contraction }\end{array}$ & $\begin{array}{l}\text { Cystoscopy injection } \\
\text { OnabotulinumtoxinA } \\
200 \mathrm{U} / 300 \mathrm{U} n=15) \text { vs. } \\
\text { Cystoscopy injection } \\
\text { with Placebo }(n=7)\end{array}$ & 1.5 & $\begin{array}{l}\text { Incontinence, urinary } \\
\text { frequency/ day, } \\
\text { nocturia/ IIQ7, UDI6, } \\
\text { pads/day, pads } \\
\text { weight/ day, MBC, } \\
\text { PVR, UTI, CIC } \\
\end{array}$ \\
\hline $\begin{array}{c}2010 \text { Dmochowski } \\
\text { [9] }\end{array}$ & N/A & $\begin{array}{l}\text { Randomized, } \\
\text { Double blinded }\end{array}$ & $\begin{array}{l}\text { OAB symptoms with } \\
\text { UUI }>6 \text { months, } \\
\text { refractory or intolerant } \\
\text { to medication }\end{array}$ & $\begin{array}{l}\text { CIC, pelvic/urological } \\
\text { abnormalities, disease related } \\
\text { bladder dysfunction }\end{array}$ & $\begin{array}{l}\text { OnabotulinumtoxinA } \\
50 \mathrm{U} / 100 \mathrm{U} / 150 \mathrm{U} / 200 \\
\mathrm{U} / 300 \mathrm{U}(n=268) \text { vs. } \\
\text { Cystoscopy injection } \\
\text { with Placebo }(n=43)\end{array}$ & 9 & $\begin{array}{l}\text { UUI, KHQ, UTI, CIC, } \\
\text { PVR > } 200 \mathrm{~mL} \text {, urine } \\
\text { retention }\end{array}$ \\
\hline 2011 Rovner [10] & N/A & $\begin{array}{l}\text { Randomized, } \\
\text { Double blinded }\end{array}$ & $\begin{array}{l}\text { OAB symptoms with } \\
\text { UUI, refractory or } \\
\text { intolerant to } \\
\text { medication }\end{array}$ & $\begin{array}{l}\text { Predominant SUI, pelvic or } \\
\text { urologic abnormality or } \\
\text { disease affect bladder } \\
\text { function, frequent UTI, PVR } \\
>200 \text {, or VV }>3000\end{array}$ & $\begin{array}{l}\text { OnabotulinumtoxinA } \\
50 \mathrm{U} / 100 \mathrm{U} / 150 \mathrm{U} / 200 \\
\mathrm{U} / 300 \mathrm{U}(n=268) \text { vs. } \\
\text { Placebo }(n=43)\end{array}$ & 9 & $\begin{array}{l}\text { UUI, urinary } \\
\text { frequency/day, Voided } \\
\text { volume, MBC, CIC, } \\
\text { PVR }>200 \mathrm{~mL}\end{array}$ \\
\hline 2012 Denys [11] & NCT 00231491 & $\begin{array}{l}\text { Randomized, } \\
\text { Double blinded }\end{array}$ & $\begin{array}{l}\text { OAB syndrome and } \\
\text { Detrusor overactivity } \\
\text { ( } \geq 3 \text { urgency/ } 3 \text { days, } \\
\text { frequency), refractory } \\
\text { or intolerant to } \\
\text { medication }\end{array}$ & $\begin{array}{c}\text { UTI, predominant SUI, PVR } \\
>150, \text { Qmax }<15, \\
\text { anticoagulation/ } \\
\text { antineoplastic or exposed to } \\
\text { OnabotulinumtoxinA }\end{array}$ & $\begin{array}{l}\text { OnabotulinumtoxinA } \\
50 \mathrm{U} / 100 \mathrm{U} / 150 \mathrm{U}(n= \\
\text { 70) vs. placebo } \\
(n=29)\end{array}$ & 6 & $\begin{array}{l}\text { Urgency, UUI, urinary } \\
\text { frequency, pads/day, } \\
\text { MBC, PVR }>50 \% \\
\text { reduction, }>75 \% \\
\text { reduction UIE, EQ-5D, } \\
\text { IQoL, UTI, CIC }\end{array}$ \\
\hline
\end{tabular}


Table 1. Cont

\begin{tabular}{|c|c|c|c|c|c|c|c|}
\hline Author, Year [ref.] & $\begin{array}{c}\text { Trial } \\
\text { Registration }\end{array}$ & Study Design & Participants & Exclusion Criteria & Group Sample & $\begin{array}{l}\text { Follow-up } \\
\text { (month) }\end{array}$ & Outcomes \\
\hline 2012 Tincello [12] & $\begin{array}{l}\text { ISRCTN } \\
26091555\end{array}$ & $\begin{array}{l}\text { Randomized, } \\
\text { Double blinded }\end{array}$ & $\begin{array}{l}\text { OAB symptoms and } \\
\text { Detrusor overactivity } \\
\text { (frequency, } \geq 2 \text { urgency } \\
\text { /day), refractory or } \\
\text { intolerant to } \\
\text { medication }\end{array}$ & $\begin{array}{l}\text { SUI, neurologic disease, } \\
\text { voiding dysfunction or } \\
\text { contraindicated to } \\
\text { OnabotulinumtoxinA }\end{array}$ & $\begin{array}{l}\text { OnabotulinumtoxinA } \\
200 \mathrm{U}(n=122) \text { vs. } \\
\text { placebo }(n=118)\end{array}$ & 3 & $\begin{array}{l}\text { Incontinence, urgency, } \\
\text { urinary frequency/day, } \\
\text { IQoL, UTI, CIC }\end{array}$ \\
\hline 2013 Chapple [13] & NCT 00910520 & $\begin{array}{l}\text { Randomized, } \\
\text { Double blinded }\end{array}$ & $\begin{array}{l}\text { OAB syndrome with } \\
\text { UUI, refractory or } \\
\text { intolerant to } \\
\text { medication in the past } \\
12 \text { months }\end{array}$ & $\begin{array}{c}\text { Previous } \\
\text { OnabotulinumtoxinA } \\
\text { treatment, with neurologic } \\
\text { reason, predominance of SUI } \\
\text { and pelvic/ urologic } \\
\text { abnormalities, bladder } \\
\text { surgery or disease affect } \\
\text { bladder function }\end{array}$ & $\begin{array}{l}\text { OnabotulinumtoxinA } \\
100 \mathrm{U}(n=277) \mathrm{vs.} \\
\text { placebo }(n=271)\end{array}$ & 6 & $\begin{array}{l}\text { Incontinence, urgency, } \\
\text { UUI, urinary } \\
\text { frequency/day, } \\
\text { nocturia, continent, } \\
\text { PVR, > 50\% reduction } \\
\text { UIE, ICIQ-SF, IUSS, } \\
\text { IQoL, UTI, CIC, }\end{array}$ \\
\hline 2011 Dowson [14] & $\begin{array}{r}\text { ISRCTN } \\
57577615\end{array}$ & $\begin{array}{l}\text { Randomized, } \\
\text { Double blinded }\end{array}$ & $\begin{array}{c}\text { Bladder } \\
\text { oversensitivity, } \\
\text { refractory or intolerant } \\
\text { to medication }\end{array}$ & $\begin{array}{c}\text { Pregnancy, breast feeding, IC, } \\
\text { neurological condition, BOO, } \\
\text { indwelling catheter, previous } \\
\text { bladder surgery, previous } \\
\text { OnabotulinumtoxinA } \\
\text { treatment, anticoagulation } \\
\text { agent use }\end{array}$ & $\begin{array}{l}\text { OnabotulinumtoxinA } \\
100 \mathrm{U}(n=10) \mathrm{vs.} \\
\text { placebo }(n=11)\end{array}$ & 3 & $\begin{array}{c}\text { Urinary } \\
\text { frequency/day, } \\
\text { Urgency, UUI, IIQ-7, } \\
\text { UDI-6, PPBC, MBC, } \\
\text { UTI, CIC }\end{array}$ \\
\hline 2013 Nitti [15] & NCT 00910845 & $\begin{array}{l}\text { Randomized, } \\
\text { Double blinded }\end{array}$ & $\begin{array}{l}\text { OAB syndrome, } \\
\text { refractory or intolerant } \\
\text { to medication }\end{array}$ & Predominance of SUI & $\begin{array}{l}\text { OnabotulinumtoxinA } \\
100 \mathrm{U}(n=278) \mathrm{vs} . \\
\text { placebo }(n=272)\end{array}$ & 3 & $\begin{array}{l}\text { Incontinence, urgency, } \\
\text { urinary frequency/day, } \\
\text { nocturia, PVR, UUI, } \\
\text { I-QoL, KHQ, UTI, CIC }\end{array}$ \\
\hline \multicolumn{8}{|c|}{ OnabotulinumtoxinA vs. PTNS } \\
\hline 2017 Sherif [16] & N/A & Randomized & $\begin{array}{l}\text { OAB symptoms, } \\
\text { refractory or intolerant } \\
\text { to medication }\end{array}$ & $\begin{array}{l}\text { Nerve damage, pregnant, } \\
\text { pacemaker, defibrillator, UTI, } \\
\text { coagulopathy, BOO, } \\
\text { neurogenic bladder, previous } \\
\text { RT or bladder cancer, s/p } \\
\text { incontinence surgery }\end{array}$ & $\begin{array}{c}\text { OnabotulinumtoxinA } \\
100 \mathrm{U}(n=30) \text { vs. } \\
\text { PTNS }(n=30)\end{array}$ & 9 & $\begin{array}{l}\text { Incontinence, urgency, } \\
\text { urinary frequency/day, } \\
\text { nocturia, OABSS, QoL, } \\
\text { frequency, nocturia, } \\
\text { PVR, Urgency scale, } \\
\text { MBC, UTI, CIC }\end{array}$ \\
\hline
\end{tabular}


Table 1. Cont.

\begin{tabular}{|c|c|c|c|c|c|c|c|}
\hline Author, Year [ref.] & $\begin{array}{c}\text { Trial } \\
\text { Registration }\end{array}$ & Study Design & Participants & Exclusion Criteria & Group Sample & $\begin{array}{l}\text { Follow-up } \\
\text { (month) }\end{array}$ & Outcomes \\
\hline \multicolumn{8}{|c|}{ PTNS vs. placebo } \\
\hline $\begin{array}{l}2010 \text { Finazzi-Agro } \\
\text { [17] }\end{array}$ & N/A & $\begin{array}{l}\text { Randomized, } \\
\text { Double blinded }\end{array}$ & $\begin{array}{l}\text { female, UI with } \\
\text { detrusor overactivity } \\
\text { incontinence, } \\
\text { refractory or intolerant } \\
\text { to medication }\end{array}$ & $\begin{array}{l}\text { Pregnancy or plan / UTI, } \\
\text { fistula, stone, Interstitial } \\
\text { cystitis, DM, pacemaker/ } \\
\text { defibrillator }\end{array}$ & $\begin{array}{l}\text { PTNS }(n=17) \text { vs. } \\
\text { placebo }(n=15)\end{array}$ & 3 & $\begin{array}{l}\text { Incontinence, urinary } \\
\text { frequency } / \text { day, } \\
\text { nocturia, }>50 \% \\
\text { reduction UIE }\end{array}$ \\
\hline 2010 Peters [18] & N/A & $\begin{array}{l}\text { Randomized, } \\
\text { Double blinded }\end{array}$ & $\begin{array}{c}\text { OAB syndrome } \\
(\mathrm{OAB}-\mathrm{q} \geq 4 \text {, voiding } \\
\geq 10 / \text { day }) \text {, refractory or } \\
\text { intolerant to } \\
\text { medication }\end{array}$ & $\begin{array}{l}\text { Pregnant or plan/ neurogenic } \\
\text { bladder/ previous use of } \\
\text { OnabotulinumtoxinA / } \\
\text { pacemaker/ defibrillator/ UTI/ } \\
\text { use of TENS }\end{array}$ & $\begin{array}{l}\text { PTNS }(n=103) \text { vs. } \\
\text { placebo }(n=105)\end{array}$ & 3 & $\begin{array}{l}\text { urinary frequency/day, } \\
\text { nocturia, OAB-qSF, } \\
\text { SF-36, GRA, voiding } \\
\text { volume, UUI }\end{array}$ \\
\hline $\begin{array}{l}2016 \text { Scaldazza } \\
\text { [19] }\end{array}$ & N/A & Randomized & $\begin{array}{l}\text { Female with } \mathrm{OAB} \\
\text { syndrome }\end{array}$ & $\begin{array}{c}\text { SUI, UTI, neurological } \\
\text { disease, bladder stone, POP, } \\
\text { pregnancy, DM, } \\
\text { anti-incontinence surgery, } \\
\text { pelvic tumor, radiation }\end{array}$ & $\begin{array}{l}\text { PTNS }(n=30) \text { vs. } \\
\text { placebo }(n=30)\end{array}$ & 3 & $\begin{array}{l}\text { urinary frequency/day, } \\
\text { voiding volume, } \\
\text { nocturia, OAB-qSF, } \\
\text { PPIUS, PGI-I }>50 \% \\
\text { reduction UIE }\end{array}$ \\
\hline \multicolumn{8}{|c|}{ SNM vs. Placebo } \\
\hline 1999 Schmidt [20] & N/A & Randomized & $\begin{array}{l}\text { UUI, poor response to } \\
\text { anti-cholinergic agents }\end{array}$ & $\begin{array}{l}\text { Neurological condition, } \mathrm{SUI} \text {, } \\
\text { pelvic pain symptoms }\end{array}$ & $\begin{array}{c}\text { SNM }(n=34) \text { vs. } \\
\text { delay SNM }(n=42)\end{array}$ & 6 & $\begin{array}{l}\text { Incontinence, } \\
\text { pads/day, }>50 \% \\
\text { reduction UIE, SF-36, } \\
\text { implant revision }\end{array}$ \\
\hline $\begin{array}{l}2000 \text { Hassouna } \\
\text { [21] }\end{array}$ & N/A & Randomized & $\begin{array}{l}\text { Urgency/ frequency } \\
\text { symptoms, refractory } \\
\text { to medication }\end{array}$ & $\begin{array}{l}\text { Neurological condition, SUI, } \\
\text { pelvic pain symptoms }\end{array}$ & $\begin{array}{l}\text { SNM }(n=25) \text { vs. no } \\
\quad \operatorname{SNM}(n=26)\end{array}$ & 6 & $\begin{array}{c}\text { Urinary } \\
\text { frequency/day, MBC, } \\
>50 \% \text { reduction UIE, } \\
\text { implant revision, } \\
\text { SF-36 }\end{array}$ \\
\hline 2000 Weil [22] & N/A & Randomized & $\begin{array}{l}\text { Refractory urinary } \\
\text { urge incontinence }\end{array}$ & $\begin{array}{c}\text { SUI, SCI, CVA within } 6 \\
\text { months, DD, bleeding } \\
\text { complication, VUR or } \\
\text { hydronephrosis, UTI, pelvic } \\
\text { pain }\end{array}$ & $\begin{array}{l}\text { SNM }(n=22) \mathrm{vs.} \\
\text { conservative } \\
\text { treatment }(n=20)\end{array}$ & 6 & $\begin{array}{l}\text { Incontinence, pad use, } \\
\text { implant revision rate, } \\
>50 \% \text { reduction UIE }\end{array}$ \\
\hline
\end{tabular}


Table 1. Cont

\begin{tabular}{|c|c|c|c|c|c|c|c|}
\hline Author, Year [ref.] & $\begin{array}{c}\text { Trial } \\
\text { Registration }\end{array}$ & Study Design & Participants & Exclusion Criteria & Group Sample & $\begin{array}{l}\text { Follow-up } \\
\text { (month) }\end{array}$ & Outcomes \\
\hline \multicolumn{8}{|c|}{ SNM vs. OnabotulinumtoxinA } \\
\hline $\begin{array}{c}2016 \text { Amundsen } \\
{[23,24]}\end{array}$ & NCT 01502956 & Randomized & $\begin{array}{l}\text { UUI, refractory or } \\
\text { intolerant to 1st and } \\
\text { 2nd line therapy }\end{array}$ & $\begin{array}{l}\text { Neurological disease, PVR } \\
>150\end{array}$ & $\begin{array}{l}\text { SNM }(n=174) \text { vs. } \\
\text { OnabotulinumtoxinA } \\
200 \mathrm{U}(n=190)\end{array}$ & 24 & $\begin{array}{c}\text { UUI, urinary } \\
\text { incontinence, pads, } \\
\text { nocturia urinary } \\
\text { frequency/day, CIC, } \\
\text { UTI, > 50\% reduction } \\
\text { UIE, Questionnaire SF, } \\
\text { Satisfaction } \\
\text { Questionnaire, PGI-I, } \\
\text { Sandvik }\end{array}$ \\
\hline
\end{tabular}

CIC: clean intermittent catheterization; OABSS: overactive bladder symptom score; UTI: urinary tract infection; I-QoL: Incontinence Quality of Life Questionnaire; IIQ-7: Incontinence Impact Questionnaire, short form; MBC: maximal bladder capacity; SUI: Stress urinary incontinence; UDI-6: Urogenital Distress Inventory, Short Form; UUI: urge urinary incontinence; $>50 \%$ reduction UIE: $>50 \%$ reduction in urinary incontinence episodes; KHQ: King's Health Questionnaire score; Questionnaire SF: Questionnaire short form; PGI-I: Patient Global Impression of Improvement; SF-36: Short Form 36 Health survey; PPIUS: Patient Perception of Intensity of Urgency Scale; OAB-qSF: Overactive bladder questionnaire short form; GRA: Global response assessment. 


\subsection{Networks}

There was sufficient evidence available for analysis of the following efficacy and safety endpoints: urinary frequency per day, incontinence episodes per day, $\geq 50 \%$ reduction of symptoms, patients with urinary tract infections (UTIs), and post-treatment urine retention needing clean intermittent catheterization (CIC). There was a lack of sufficient data to make comparisons between the three treatment modalities with regard to the QoL, urgency, urge incontinence episodes/day, maximal bladder capacity, and nocturia. Results of the pair-wise comparison meta-analyses are shown in Table 2.

Table 2. Pairwise meta-analyses result for different endpoints.

\begin{tabular}{|c|c|c|c|c|c|}
\hline Endpoint & Comparison & $N$ & $I^{2}(\%)$ & $p$ Value & $\begin{array}{c}\text { Standard Mean Difference } \\
(95 \% \mathrm{CI})\end{array}$ \\
\hline \multirow{4}{*}{$\begin{array}{l}\text { Urinary } \\
\text { frequency/day }\end{array}$} & $\begin{array}{l}\text { OnabotulinumtoxinA vs. } \\
\text { Placebo }\end{array}$ & 4 & 92 & $<0.001$ & $-0.65(-0.24--1.06)$ \\
\hline & $\begin{array}{c}\text { PTNS vs. } \\
\text { OnabotulinumtoxinA }\end{array}$ & 1 & \multirow{3}{*}{37.1} & \multirow{3}{*}{0.204} & $-1.02(-1.55--0.48)$ \\
\hline & PTNS vs. Placebo & 3 & & & $-0.37(-0.03--0.70)$ \\
\hline & SNM vs. Placebo & 1 & & & $-1.12(-0.53--1.71)$ \\
\hline $\begin{array}{l}\text { Urge urine } \\
\text { incontinence }\end{array}$ & $\begin{array}{l}\text { OnabotulinumtoxinA vs. } \\
\text { Placebo }\end{array}$ & 2 & 70.7 & 0.065 & $-0.37(-0.05-0.79)$ \\
\hline $\begin{array}{l}\text { Urgency } \\
\text { Episode }\end{array}$ & $\begin{array}{l}\text { OnabotulinumtoxinA vs. } \\
\text { Placebo }\end{array}$ & 4 & 97.6 & $<0.001$ & $-0.84(-0.08-1.60)$ \\
\hline \multirow{2}{*}{ Maximal } & PTNS vs. Placebo & 1 & & & $1.35(0.79-1.92)$ \\
\hline & SNM vs. Placebo & 1 & & & $0.91(0.33-1.48)$ \\
\hline \multirow[t]{2}{*}{ I-QoL } & $\begin{array}{l}\text { OnabotulinumtoxinA vs. } \\
\text { Placebo }\end{array}$ & 2 & \multirow[t]{2}{*}{99.1} & \multirow[t]{2}{*}{$<0.001$} & $0.98(-0.89-2.86)$ \\
\hline & PTNS vs. Placebo & 1 & & & $0.86(0.13-1.59)$ \\
\hline \multirow{4}{*}{ Incontinence } & $\begin{array}{l}\text { OnabotulinumtoxinA vs. } \\
\text { Placebo }\end{array}$ & 3 & 97.8 & $<0.001$ & $-0.84(-1.62-0.06)$ \\
\hline & $\begin{array}{c}\text { PTNS vs. } \\
\text { OnabotulinumtoxinA }\end{array}$ & 1 & \multirow[b]{3}{*}{74.6} & & $0.54(0.02-1.06)$ \\
\hline & PTNS vs. Placebo & 1 & & & $-1.49(-2.28-0.70)$ \\
\hline & SNM vs. Placebo & 2 & & 0.047 & $-2.10(-3.07-1.12)$ \\
\hline \multirow{4}{*}{$\begin{array}{c}\geq 50 \% \\
\text { Improvement }\end{array}$} & $\begin{array}{c}\text { Placebo vs. } \\
\text { OnabotulinumtoxinA }\end{array}$ & 2 & 0.0 & 0.410 & $0.53(0.40-0.70)$ \\
\hline & $\begin{array}{c}\text { PTNS vs. } \\
\text { OnabotulinumtoxinA }\end{array}$ & 2 & 0.0 & 0.371 & $0.50(0.32-0.76)$ \\
\hline & Placebo vs. PTNS & 3 & \multirow[t]{2}{*}{52.5} & \multirow[t]{2}{*}{0.122} & $0.21(0.07-0.61)$ \\
\hline & SNM vs. Placebo & 1 & & & $1.27(0.87-1.87)$ \\
\hline \multirow{3}{*}{$\begin{array}{l}\text { Urinary tract } \\
\text { infection }\end{array}$} & $\begin{array}{l}\text { OnabotulinumtoxinA vs. } \\
\text { Placebo }\end{array}$ & 8 & \multirow[t]{3}{*}{0} & \multirow[t]{3}{*}{0.486} & $2.55(1.89-3.43)$ \\
\hline & $\begin{array}{c}\text { PTNS vs. } \\
\text { OnabotulinumtoxinA }\end{array}$ & 1 & & & $0.20(0.01-4.34)$ \\
\hline & $\begin{array}{c}\text { SNM vs. } \\
\text { OnabotulinumtoxinA }\end{array}$ & 1 & & & $0.33(0.19-0.56)$ \\
\hline \multirow{3}{*}{$\begin{array}{c}\text { Clean } \\
\text { intermittent } \\
\text { catherization }\end{array}$} & $\begin{array}{l}\text { OnabotulinumtoxinA vs. } \\
\text { Placebo }\end{array}$ & 9 & \multirow[t]{3}{*}{0} & \multirow[t]{3}{*}{0.786} & $5.95(3.08-11.46)$ \\
\hline & $\begin{array}{c}\text { PTNS vs. } \\
\text { OnabotulinumtoxinA }\end{array}$ & 1 & & & $0.20(0.01-4.34)$ \\
\hline & $\begin{array}{c}\text { SNM vs. } \\
\text { OnabotulinumtoxinA }\end{array}$ & 1 & & & $0.01(0.00-0.23)$ \\
\hline
\end{tabular}

\subsection{Risk of Bias Assessment}

A summary of the included studies and a risk of bias graph are shown in Figure 2. The four studies that compared SNM with delayed SNM were rated as having a high risk of bias in the "measurement of outcome' category because the self-reporting results could have been influenced by the placebo effect. As these papers did not describe the randomization method or specify whether the assessors were blinded, we had some concerns regarding the randomization process when the risk of bias was 
evaluated. All of the studies, except the four that compared the efficacy of SNM with the delayed SNM group, were judged as having a 'low risk' of bias or as having 'some concerns'.

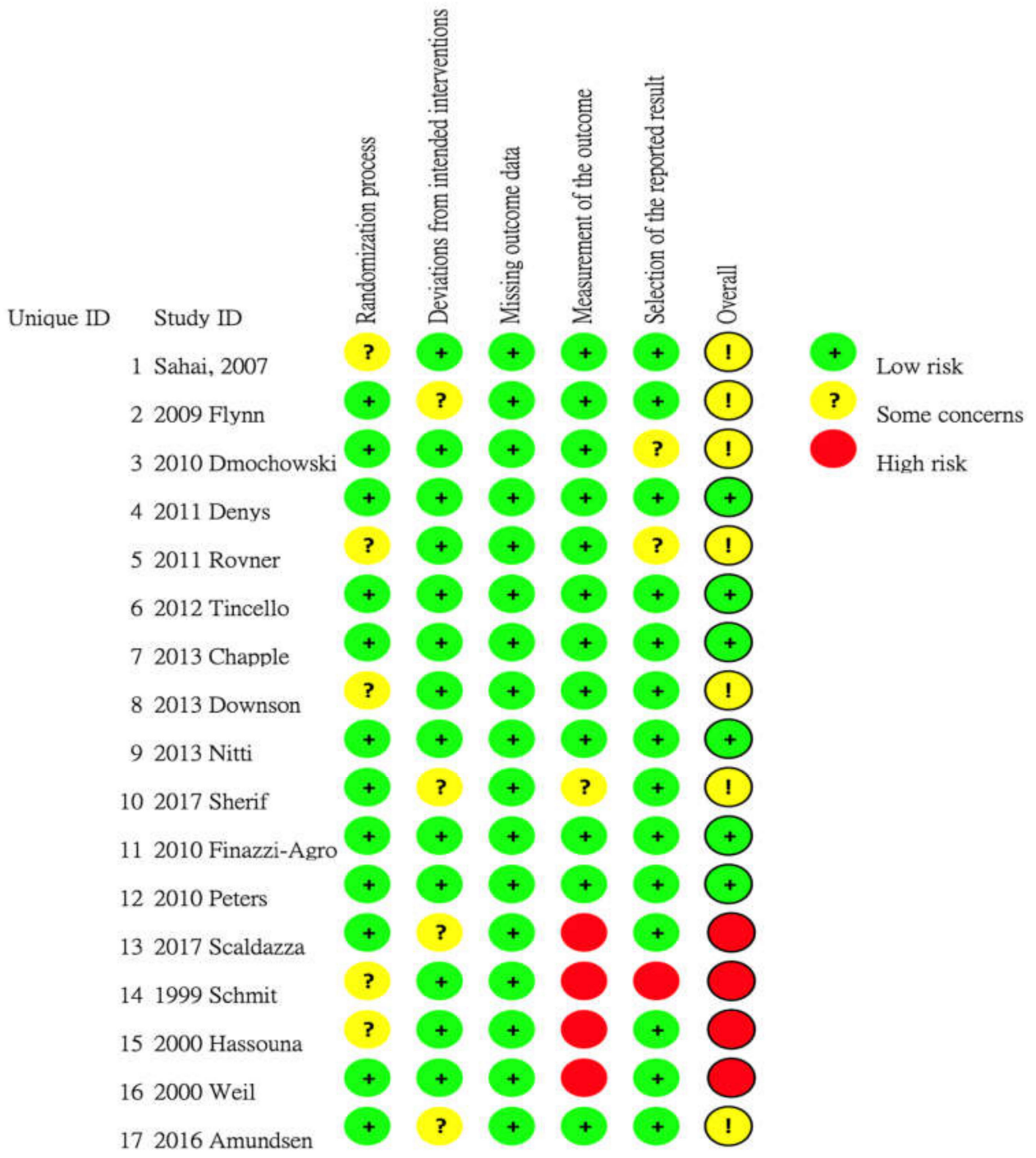

Figure 2. Risk of bias graph and summary of the included studies: Reviewers' judgments regarding each risk of bias item for the included studies. The figure was generated using RoB 2 tool (the 22 August 2019 version)

\subsection{Network Meta-Analysis on the Outcomes of Interests}

\subsubsection{Efficacy}

\section{Urinary Frequency per Day}

A total of nine studies contributed to the comparison of urine frequency per day [6-14]. Pair-wise comparisons with a random effects (RE) model revealed that all three modalities were more efficacious than the placebo (Table 2). The NMA (Network Meta-analysis) identified a greater reduction in the total number of micturition per day for SNM compared with the placebo, PTNS, and OnabotulinumtoxinA. There were no significant differences observed between OnabotulinumtoxinA and SNM (Table 3). The ranking probability results are shown in Figure 3. The ranking results for urinary frequency reduction was as follows: SNM ranked first, OnabotulinumtoxinA ranked second, PTNS ranked third, and placebo ranked fourth (Figure 3A). 
Table 3. Summary of results from NMA (on the lower triangle) and traditional pairwise meta-analysis (on the upper triangle).

\begin{tabular}{|c|c|c|c|c|}
\hline & Placebo & Onabotulinumtoxin & PTNS & SNM \\
\hline \multicolumn{5}{|c|}{ Urinary frequency/ Day (SMD, 95\% CI) } \\
\hline Placebo & 0 & $-0.65(-0.24-1.06)$ & $-0.37(-0.03-0.70)$ & $-1.12(-0.53-1.71)$ \\
\hline OnabotulinumtoxinA & $-1.72(-1.23-2.21)$ & 0 & $-1.02(-1.55--0.48)$ & \\
\hline PTNS & $-0.80(-0.15-1.14)$ & $-0.92(-1.59--0.26)$ & 0 & \\
\hline SNM & $-8.10(-4.04-12.16)$ & $-6.38(-2.29-10.47)$ & $\begin{array}{c}-7.30 \\
(-3.19-11.41)\end{array}$ & 0 \\
\hline \multicolumn{5}{|c|}{ Incontinence/ Day (SMD, 95\% CI) } \\
\hline Placebo & 0 & $-0.84(-0.06-1.62)$ & $-1.49(-0.70-2.28)$ & $-2.10(-1.12-3.07)$ \\
\hline OnabotulinumtoxinA & $-1.96(-0.92-3.00)$ & 0 & $-0.54(-0.03-1.06)$ & \\
\hline PTNS & $-2.05(-0.56-3.53)$ & $-0.08(-1.37-1.53)$ & 0 & \\
\hline SNM & $\begin{array}{c}-10.96 \\
(-8.60-13.31)\end{array}$ & $\begin{array}{c}-8.99 \\
(-6.42-11.57)\end{array}$ & $-8.91(-6.12-11.70)$ & 0 \\
\hline \multicolumn{5}{|c|}{ Urinary tract infection $(\mathrm{OR}, 95 \% \mathrm{CI})$} \\
\hline Placebo & 1 & $2.54(1.89-3.44)$ & & \\
\hline OnabotulinumtoxinA & $3.06(2.26-4.15)$ & 1 & $0.20(0.01-4.35)$ & $0.33(0.19-0.56)$ \\
\hline PTNS & $0.57(0.03-12.62)$ & $0.19(0.01-4.06)$ & 1 & \\
\hline SNM & $10.73(0.39-1.38)$ & $0.24(0.14-0.42)$ & $1.28(0.06-29.29)$ & 1 \\
\hline \multicolumn{5}{|c|}{ Clean intermittent catheterization (OR, 95\% CI) } \\
\hline Placebo & 1 & $5.95(3.08-11.46)$ & & \\
\hline OnabotulinumtoxinA & $6.92(3.18-15.06)$ & 1 & $0.20(0.01-4.34)$ & $0.01(0.00-0.23)$ \\
\hline PTNS & $1.29(0.05-31.93)$ & $0.19(0.01-4.19)$ & 1 & \\
\hline SNM & $0.08(0.00-1.46)$ & $0.01(0.00-0.19)$ & $0.06(0.00-4.01)$ & 1 \\
\hline
\end{tabular}
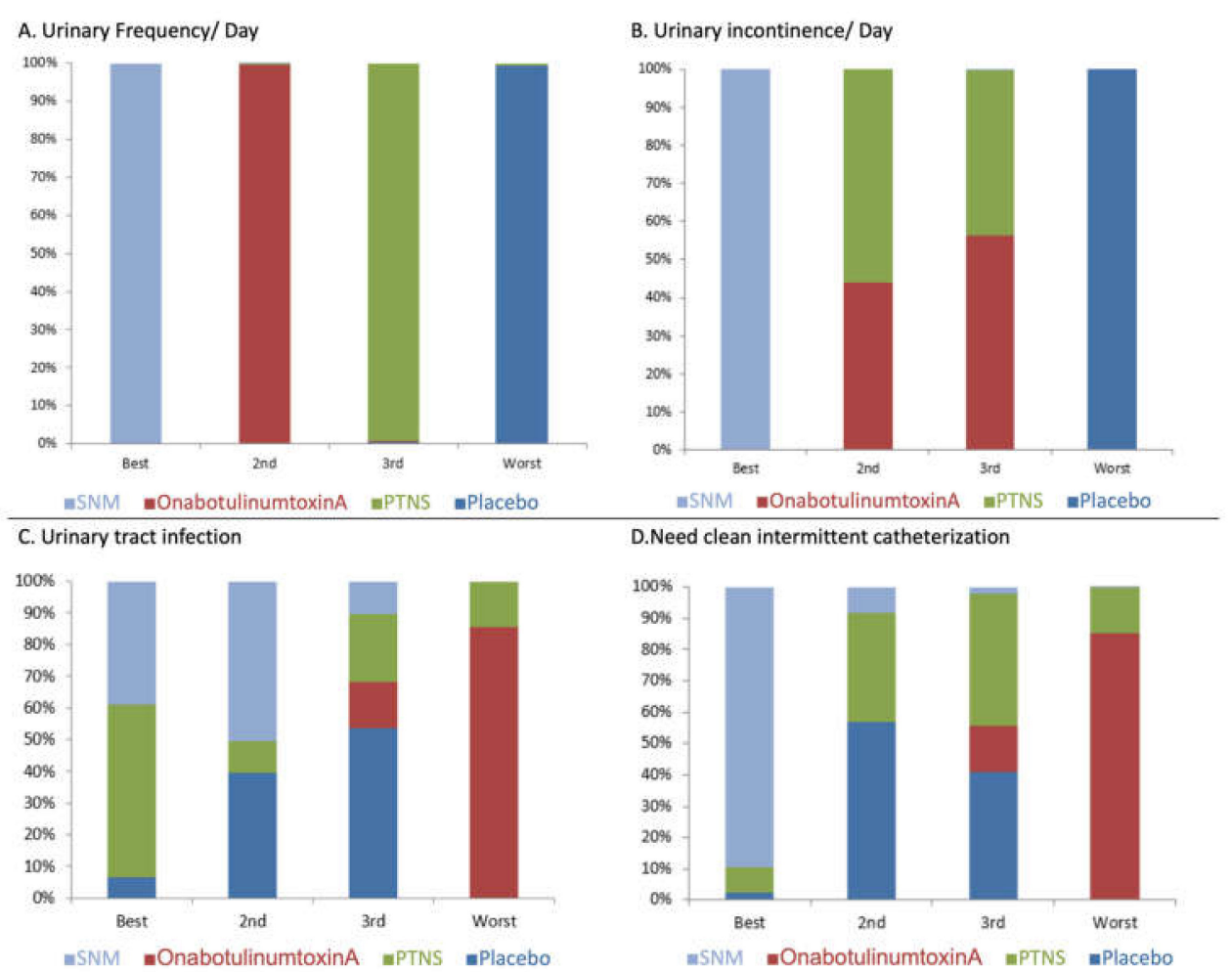

D.Need clean intermittent catheterization

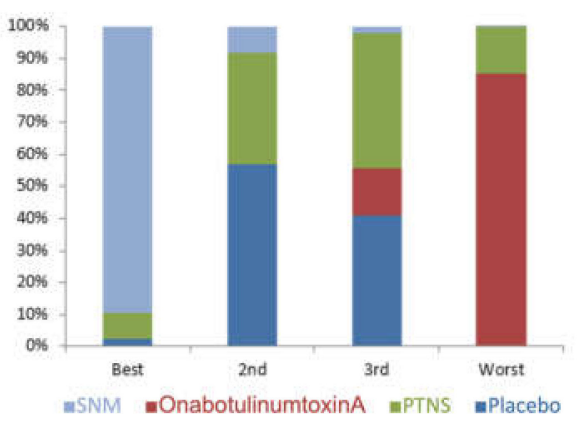

Figure 3. Treatment rankings for (A) urinary frequency/day, (B) incontinence, (C) urinary tract infection, and $(\mathbf{D})$ urine retention needing clean intermittent catheterization. 
Urinary Incontinence Episodes per Day

There were seven studies used to compare the efficacy of the three modalities on the number of incontinence episodes per day at 12 weeks follow-up [12,13,15-17,20,22]. Pair-wise comparisons with a RE model revealed that all three modalities were more efficacious than the placebo (Table 2). The NMA demonstrated that SNM was associated with a greater reduction in the total number of incontinence episodes per day compared with the placebo, PTNS, and OnabotulinumtoxinA. There was no significant difference between the efficacy of OnabotulinumtoxinA and PTNS (Table 3). The ranking results for incontinence episode reduction was as follows: SNM ranked first, PTNS ranked second, OnabotulinumtoxinA ranked third, and placebo ranked fourth (Figure 3B).

\section{$\geq 50 \%$ Symptom Improvement at 12 Weeks Follow-up}

The network of eligible comparisons for $>50 \%$ symptom improvement is shown in Figure $4 \mathrm{D}$. There were eight studies that reported parameters including $\geq 50 \%$ improvement in symptoms at 12 weeks follow-up. Pair-wise comparisons with a RE model revealed all three modalities were more efficacious than the placebo. However, there was a significant inconsistency expected in $\geq 50 \%$ reduction of symptoms improvement. Therefore, network meta-analysis and ranking probability was not conducted with regard to this parameter.

A. Urinary frequency/ Day

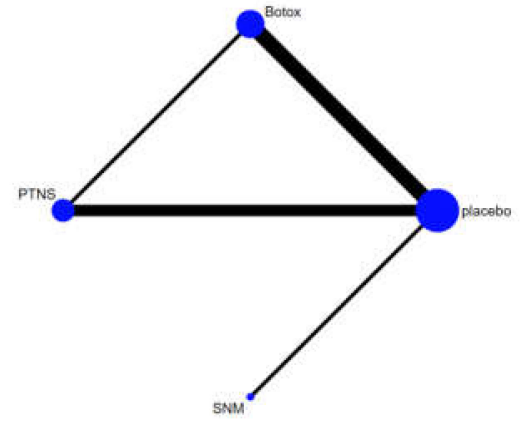

C. Urinary tract infection

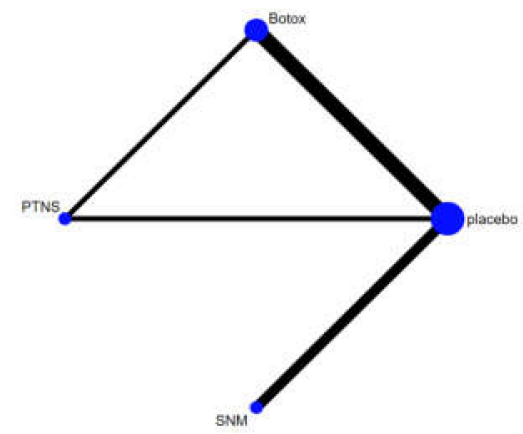

B. Incontinence/ Day

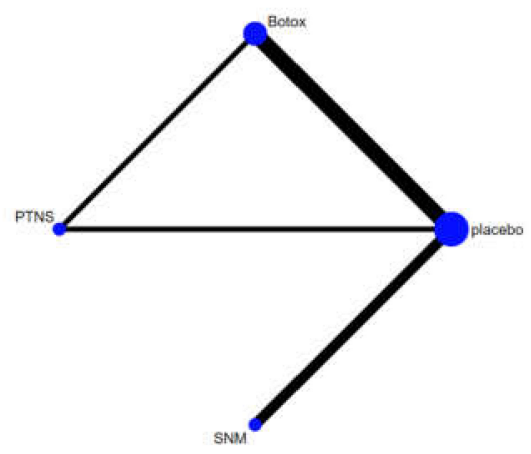

D. $50 \%$ of symptoms improvement

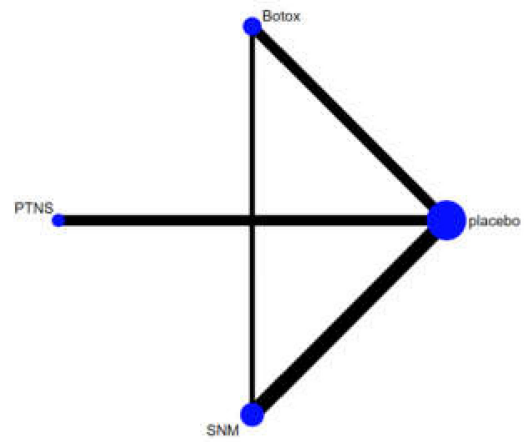

Figure 4. Network of treatment comparisons between OnabotulinumtoxinA (Botox), sacral neuromodulation (SNM), percutaneous tibial nerve stimulation (PTNS) in regard to (A) urinary frequency/day, (B) incontinence, (C) urinary tract infection, and (D) $\geq 50 \%$ of symptoms improvement. The figure was generated using R 3.3.2 software. 


\subsection{Complications}

\subsubsection{Urinary Tract Infection}

Estimates of the treatment effectiveness on the occurrence of UTIs were informed by 10 studies [7-9,11-16,23,24]. The RE model revealed that OnabotulinumtoxinA was associated with a higher incidence of UTIs compared with the placebo, SNM, and PTNS (Table 3). The ranking results for the post-management risk of UTIs was as follows: PTNS ranked best, SNM ranked second, placebo ranked third, and OnabotulinumtoxinA ranked worst (Figure 3C).

\subsubsection{Urine Retention Needing Clean Intermittent Catheterization}

Estimates on the effect of treatments on post-management urine retention were reported in 11 studies $[7-16,23,24]$. The RE model demonstrated that OnabotulinumtoxinA was associated with a higher occurrence of post-treatment urine retention needing catherization compared with the placebo, SNM, and PTNS (Table 3). The ranking results for post-management risk of post-treatment urine retention was as follows: SNM ranked best, placebo ranked second, PTNS ranked third, and OnabotulinumtoxinA ranked worst (Figure 3D).

\section{Discussion}

The AUA guidelines suggest intra-detrusor injection with OnabotulinumtoxinA (evidence grade B), SNM (evidence grade C), or PTNS (evidence grade C) as the third-line treatment modalities for adult $\mathrm{OAB}$ [3]. In the absence of a direct head to head comparison of the three treatment modalities for adult OAB symptoms, the present detailed systemic review and network meta-analysis is the first review to combine all updated evidence and compares the efficacy of any dose of OnabotulinumtoxinA, sacral neuromodulation and PTNS. The compared outcomes included voiding frequency/day, urinary incontinence episodes/day, and $\geq 50 \%$ reduction in symptoms. Pairwise meta-analysis revealed that all three modalities were more efficacious than a placebo with regard to the outcomes of interests, including urinary frequency, incontinence, and achieving $\geq 50 \%$ of symptoms improvement. SNM achieved the greatest reduction in urinary incontinence episodes and voiding frequency/day. OnabotulinumtoxinA was associated with the highest risk of urine retention and UTI episodes in the follow-up period. As none of the included studies used a unified or standard questionnaire to evaluate the QoL, the results regarding QoL were not pooled for the meta-analysis. We suggested that International Continence Society or International Urogynecology Association should unify the QoL questionnaire based on evidence and experts' opinion for a better evaluation of post treatment result.

The cost of the treatment and the insurance payment system may influence the patient's preference for a specific therapy. A cost analysis was performed to assess the economic effectiveness of each treatment. Based on a literature review, SNM was considered to be the most expensive treatment compared with a OnabotulinumtoxinA injection and PTNS in the short term [25-27]. However, in a model of middle- and long-term treatment, the cost-effectiveness of SNM was comparable with OnabotulinumtoxinA $[25,28]$. There was no comparison between OnabotulinumtoxinA and PTNS. Martinson et al. constructed the Markov model to simulate the cost-effectiveness of PTNS and they concluded that PTNS was the least costly therapy compared with OnabotulinumtoxinA and SNM [26]. However, different regional health insurance and health care payment systems could affect the simulation result and lead to different outcomes. A local cost-effectiveness analysis is more valuable for urologists.

The current study primarily compared short-term efficacy at 12 weeks follow-up and data comparing long-term efficacy is lacking. Amundsen et al. conducted a randomized control trial with a 6 years follow-up, which confirmed the middle- to long-term efficacy, QoL and satisfaction with treatment for OnabotulinumtoxinA injection and SNM [24]. However, there has been no previous report on the long-term efficacy of PTNS. The present study did not compare long-term adherence between treatment modalities. 
The major drawbacks for management with antimuscarinics lie in the low rates of adherence to the medication [29,30]. Adherence at 12 months was $39 \%$ for mirabegron vs. $14-35 \%$ for antimuscarinics [29]. The effects of OnabotulinumtoxinA persist for 6-9 months and the effect can be extended with repeated injections [31]. Long-term adherence to OnabotulinumtoxinA injections is less discussed. Patient preference is an important factor, especially when comprehensive data is not available to assist in the decision-making progress. No significant difference in patient satisfaction was reported between SNM and OnabotulinumtoxinA in the ROSETTA trials [23,24] or studies by Hoag et al. [32]. Since all three of the investigated third line treatments are effective, health care providers should carefully discuss the pros and cons of each treatment with the patient and determine the appropriate strategy based on each individual situation.

The adverse effects of an OnabotulinumtoxinA injection include hematuria, bacteriuria, UTIs, urine retention, and increased post-void residual urine [33]. The rate of post-therapeutic complications, including urine retention that needed clean intermittent catheterization and UTIs, were compared between treatments. Intravesical injection with OnabotulinumtoxinA lead to a significantly higher rate of CIC and UTIs. Nevertheless, the higher rate of CIC was not consistent among the included trials and the literature review, therefore this conclusion could be controversial. Side effects associated with SNM included pain at the stimulator and lead sites, lead migration, infection, and the requirement for surgical revision [21-24,32]. A screen test before implantation and two-stage implantation could increase the success rate [34]. The revision rate varied from 3-32\% and the removal rate varied from $8.6-13 \%[24,35]$. With improvements in battery longevity and better localization of lead placement, these revision and removal rates could be reduced. The only side effects of PTNS were local adverse events, including minor bleeding spots and temporary pain [4].

There were several limitations to the current network meta-analysis study. First, the variable qualities and publication biases of the included studies may compromise the results of the network meta-analysis. There was considerable heterogeneity across the study designs, including participants, scheduled follow-ups, questionnaires, evaluated parameters, OnabotulinumtoxinA dose, and SNM and PTNS protocols. The U.S Food and Drug Administration approved intra-detrusor injection of OnabotulinumtoxinA is $100 \mathrm{U}$ [3], while the included ROSETTA trial used $200 \mathrm{U}$ [23,24]. Different protocols were also used for PTNS. Second, the variable or ambiguous and potentially post-hoc definitions for the outcome parameters used in each study made it difficult to unify and compare the treatment results between the studies. However, because of the heterogeneity of enrolled patients, existing of high-risk bias and lacking long-term outcomes comparison, more high-quality studies are necessary to clarify this benefit and risk of the current available 3rd line therapy for overactive bladder symptoms. A strength of the current study was that it collected 17 randomized controlled trials published in English over three decades and used network meta-analysis to compare the three existing therapeutic options.

\section{Conclusions}

The results revealed that all three modalities were efficacious in managing adult OAB syndrome, and all were better than a placebo on the specific symptoms reported to be the outcome of the study. This review shows that at 12 weeks follow-up, SNM yielded the greatest reduction in urinary incontinence episodes and urinary frequency/day. OnabotulinumtoxinA resulted in a higher incidence of complications, including urinary tract infection and urinary retention.

As there is a lack of head to head comparison studies among SNM, PTNS, and OnabotulinumtoxinA for the treatment of adult $\mathrm{OAB}$ symptoms, the current network meta-analysis represents the best available evidence for the comparison of these three treatment modalities. 


\section{Material and Methods}

\subsection{Search Strategy and Eligibility Criteria}

To identify appropriate studies for a network meta-analysis, two independent investigators (CWL and SJC) conducted a comprehensive electronic literature search of PubMed, EMBASE, Cochrane Central Register of Controlled Trials (CENTRAL), Wiley, and ClinicalTrial.gov for trials published between January 1995 and September 2019, with a language restriction of English. The present study followed PRISMA recommendations. The terms and related synonyms "overactive bladder", "bladder overactivity", "detrusor overactivity", "urinary incontinence", "urgency", "urgent incontinence", "detrusor overactivity" and "Botulinum A toxin" or "OnabotulinumtoxinA" or "Botox" or "botulinumtoxin A" or "sacral neuromodulation" or "sacral nerve stimulation" or "percutaneous tibial nerve stimulation" or "posterior tibial nerve stimulation" were combined in the search strategy. $\mathrm{MeSH}$ terms, key words and other free terms were used for searching and Boolean operators (OR and AND) were used to combine the searches. The reference lists of the included articles, as well as the guidelines for the AUA and European Association of Urology were manually reviewed, and external peer reviewers were asked to contribute any additional trials.

Studies were considered eligible if they were randomized controlled trials that compared any dose of OnabotulinumtoxinA, SNM, and PTNS therapy with each other or a placebo, in adults with $\mathrm{OAB}$ syndrome with reported efficacy at 12 weeks follow-up. It was required that the studies provided detailed data on the treatments and outcomes of the participants.

\subsection{Data Extraction and Quality Asessment}

Two investigators (CWL and SJC) independently reviewed the titles and abstracts to check their relevance and adherence to the eligibility criteria. The full text of articles was assessed if their eligibility was not clear from the abstract. A preliminary network was constructed based on the intervention and comparators in the included trials. The homogeneity of the included trials was also evaluated.

Two investigators (CWL and SJC) reviewed the quality of the included studies. The risk of bias was evaluated using the RoB 2 (Version 2 of the Cochrane risk-of-bias tool for randomized trials) tool to evaluate the quality of evidence [36]. The following domains were evaluated: random sequence generation, allocation concealment, blinding, incomplete data on outcomes, selective reporting, and other bias. The Grading of Recommendation, Assessment, Development, and Evaluation Working Group approach for rating the quality of treatment effect estimates was also followed. Urination frequency/day, incontinence, and $\geq 50 \%$ symptom improvement were selected because of their relevance to clinical symptom improvement. The quality of evidence was rated as very low, low, moderate or high.

When standard deviation data was missing or only $95 \%$ confidence intervals (CI) were listed, the standard deviation was calculated using the formula in the Cochrane handbook for systematic reviews of interventions, or it was calculated from the figure data in the article or supplemental data.

\subsection{Network Development}

If the data was available, theoretical networks were developed for each outcome based on the similarities between studies. The outcomes included QoL, lower urinary tract symptoms at 12 weeks follow-up, including urinary incontinence episodes, urgency episodes, urge urinary incontinence episodes/day and urinary frequency/day, achieving $\geq 50 \%$ of symptoms improvement, nocturia, and complications including post-treatment urine retention that needed intermittent catheterization and urinary tract infections.

\subsection{Statistical Analysis}

The network meta-analysis was performed using R 3.3.2 software (Bell Laboratories, Madison, WI, USA, 2016) and STATA version 15.0 (StataCorp. LP, College Station, TX, USA, 2017). All outcomes 
of interests were compared pairwise by calculating $I^{2}$ statistics. Study heterogeneity was assessed using the R package. Node splitting analysis was performed to evaluate inconsistencies by comparing differences between the direct and indirect evidence. Dichotomous variables and continuous variables were expressed as odds ratios (ORs) with 95\% CIs and weighted mean differences with $95 \%$ CIs, respectively. A RE model was used to calculate evidence inconsistencies because of the existence of heterogeneity among the included trials and each intervention comparison. The ranking probabilities for the different $\mathrm{OAB}$ symptoms interventions were also calculated with regard to each outcome of interest. Additionally, publication bias was evaluated according to the symmetry characteristics of funnel plots, with a symmetrical and concentrated distribution of dots implying no significant deviation.

Author Contributions: Conceptualization, S.-J.C.; methodology, M.-Y.W.; software, M.-Y.W.; validation, C.-W.L. and S.-J.C.; formal analysis, M.-Y.W.; investigation, C.-W.L.; data curation, C.-W.L.; writing-original draft preparation, C.-W.L.; writing-review and editing, S.-J.C.; visualization, C.-W.L.; supervision, F.-S.J. and S.S.-D.Y. All authors have read and agree to the published version of the manuscript.

Funding: This research was funded by Taipei Tzu Chi Hospital, Buddhist Tzu Chi Medical Foundation, grant number: TCRD-TPE-106-RT-6 and TCRD-TPE-107-51

Conflicts of Interest: All contributing authors declare that they have no conflicting interests.

\section{References}

1. Abrams, P.; Cardozo, L.; Fall, M.; Griffiths, D.; Rosier, P.; Ulmsten, U.; Van Kerrebroeck, P.; Victor, A.; Wein, A.; Standardisation Sub-Committee of the International Continence Society. The standardisation of terminology of lower urinary tract function: Report from the standardisation sub-committee of the international continence society. Neurourol. Urodyn. 2002, 21, 167-178. [CrossRef] [PubMed]

2. Irwin, D.E.; Kopp, Z.S.; Agatep, B.; Milsom, I.; Abrams, P. Worldwide prevalence estimates of lower urinary tract symptoms, overactive bladder, urinary incontinence and bladder outlet obstruction. BJU Int. 2011, 108, 1132-1138. [CrossRef] [PubMed]

3. Lightner, D.J.; Gomelsky, A.; Souter, L.; Vasavada, S.P. Diagnosis and treatment of overactive bladder (Non-Neurogenic) in adults: AUA/SUFU guideline amendment 2019. J. Urol. 2019, 202, 558-563. [CrossRef] [PubMed]

4. Niu, H.L.; Ma, Y.H.; Zhang, C.J. Comparison of OnabotulinumtoxinA versus sacral neuromodulation for refractory urinary urge incontinence: A systematic review and meta-analysis of randomized controlled trials. Int. J. Surg. 2018, 60, 141-148. [CrossRef] [PubMed]

5. Arruda, R.M.; Takano, C.C.; Girão, M.J.B.C.; Haddad, J.M.; Aleixo, G.F.; Castro, R.A. Treatment of non-neurogenic overactive bladder with OnabotulinumtoxinA: Systematic review and meta-analysis of prospective, randomized, placebo-controlled clinical trials. Rev. Bras. Ginecol. Obstet. 2018, 40, 225-231. [CrossRef]

6. Moher, D.; Liberati, A.; Tetzlaff, J.; Altman, D.G.; The PRISMA Group. Preferred Reporting Items for Systematic Reviews and Meta-Analyses: The PRISMA Statement. Ann. Intern. Med. 2009, 151, 264-269. [CrossRef]

7. Sahai, A.; Khan, M.S.; Dasgupta, P. Efficacy of botulinum toxin-A for treating idiopathic detrusor overactivity: Results from a single center, randomized, double-blind, placebo controlled trial. J. Urol. 2007, 177, 2231-2236. [CrossRef]

8. Flynn, M.K.; Amundsen, C.L.; Perevich, M.; Liu, F.; Webster, G.D. Outcome of a randomized, double-blind, placebo controlled trial of botulinum A toxin for refractory overactive bladder. J. Urol. 2009, 181, 2608-2615. [CrossRef]

9. Dmochowski, R.; Chapple, C.; Nitti, V.W.; Chancellor, M.; Everaert, K.; Thompson, C.; Daniell, G.; Zhou, J.; Haag-Molkenteller, C. Efficacy and safety of onabotulinumtoxinA for idiopathic overactive bladder: A double-blind, placebo controlled, randomized, dose ranging trial. J. Urol. 2010, 184, 2416-2422. [CrossRef]

10. Rovner, E.; Kennelly, M.; Schulte-Baukloh, H.; Zhou, J.; Haag-Molkenteller, C.; Dasgupta, P. Urodynamic results and clinical outcomes with intradetrusor injections of onabotulinumtoxinA in a randomized, placebo-controlled dose-finding study in idiopathic overactive bladder. Neurourol. Urodyn. 2011, 30, 556-562. [CrossRef] [PubMed] 
11. Denys, P.; Le Normand, L.; Ghout, I.; Costa, P.; Chartier-Kastler, E.; Grise, P.; Hermieu, J.F.; Amarenco, G.; Karsenty, G.; Saussine, C.; et al. Efficacy and safety of low doses of onabotulinumtoxinA for the treatment of refractory idiopathic overactive bladder: A multicentre, double-blind, randomised, placebo-controlled dose-ranging study. Eur. Urol. 2012, 61, 520-529. [CrossRef] [PubMed]

12. Tincello, D.G.; Kenyon, S.; Abrams, K.R.; Mayne, C.; Toozs-Hobson, P.; Taylor, D.; Slack, M. Botulinum toxin a versus placebo for refractory detrusor overactivity in women: A randomised blinded placebo-controlled trial of 240 women (the RELAX study). Eur. Urol. 2012, 62, 507-514. [CrossRef] [PubMed]

13. Chapple, C.; Sievert, K.D.; MacDiarmid, S.; Khullar, V.; Radziszewski, P.; Nardo, C.; Thompson, C.; Zhou, J.; Haag-Molkenteller, C. OnabotulinumtoxinA 100 U significantly improves all idiopathic overactive bladder symptoms and quality of life in patients with overactive bladder and urinary incontinence: A randomised, double-blind, placebo-controlled trial. Eur. Urol. 2013, 64, 249-256. [CrossRef] [PubMed]

14. Dowson, C.; Sahai, A.; Watkins, J.; Dasgupta, P.; Khan, M.S. The safety and efficacy of botulinum toxin-A in the management of bladder oversensitivity: A randomised double-blind placebo-controlled trial. Int. J. Clin. Pract. 2011, 65, 698-704. [CrossRef]

15. Nitti, V.W.; Dmochowski, R.; Herschorn, S.; Sand, P.; Thompson, C.; Nardo, C.; Yan, X.; Haag-Molkenteller, C.; EMBARK Study Group. OnabotulinumtoxinA for the treatment of patients with overactive bladder and urinary incontinence: Results of a phase 3, randomized, placebo controlled trial. J. Urol. 2013, 189, 2186-2193. [CrossRef]

16. Sherif, H.; Khalil, M.; Omar, R. Management of refractory idiopathic overactive bladder: Intradetrusor injection of botulinum toxin type A versus posterior tibial nerve stimulation. Can. J. Urol. 2017, 24, 8838-8846.

17. Finazzi-Agro, E.; Petta, F.; Sciobica, F.; Pasqualetti, P.; Musco, S.; Bove, P. Percutaneous tibial nerve stimulation effects on detrusor overactivity incontinence are not due to a placebo effect: A randomized, double-blind, placebo controlled trial. J. Urol. 2010, 184, 2001-2006. [CrossRef]

18. Peters, K.M.; Carrico, D.J.; Perez-Marrero, R.A.; Khan, A.U.; Wooldridge, L.S.; Davis, G.L.; Macdiarmid, S.A. Randomized trial of percutaneous tibial nerve stimulation versus Sham efficacy in the treatment of overactive bladder syndrome: Results from the SUmiT trial. J. Urol. 2010, 183, 1438-1443. [CrossRef]

19. Scaldazza, C.V.; Morosetti, C.; Giampieretti, R.; Lorenzetti, R.; Baroni, M. Percutaneous tibial nerve stimulation versus electrical stimulation with pelvic floor muscle training for overactive bladder syndrome in women: Results of a randomized controlled study. Int. Braz. J. Urol. 2017, 43, 121-126. [CrossRef]

20. Schmidt, R.A.; Jonas, U.; Oleson, K.A.; Janknegt, R.A.; Hassouna, M.M.; Siegel, S.W.; van Kerrebroeck, P.E. Sacral nerve stimulation for treatment of refractory urinary urge incontinence. Sacral Nerve Stimulation Study Group. J. Urol. 1999, 162, 352-357. [CrossRef]

21. Hassouna, M.M.; Siegel, S.W.; Nyeholt, A.A.; Elhilali, M.M.; van Kerrebroeck, P.E.; Das, A.K.; Gajewski, J.B.; Janknegt, R.A.; Rivas, D.A.; Dijkema, H.; et al. Sacral neuromodulation in the treatment of urgency-frequency symptoms: A multicenter study on efficacy and safety. J. Urol. 2000, 163, 1849-1854. [CrossRef]

22. Weil, E.H.; Ruiz-Cerda, J.L.; Eerdmans, P.H.; Janknegt, R.A.; Bemelmans, B.L.; van Kerrebroeck, P.E. Sacral root neuromodulation in the treatment of refractory urinary urge incontinence: A prospective randomized clinical trial. Eur. Urol. 2000, 37, 161-171. [CrossRef]

23. Amundsen, C.L.; Richter, H.E.; Menefee, S.A.; Komesu, Y.M.; Arya, L.A.; Gregory, W.T.; Myers, D.L.; Zyczynski, H.M.; Vasavada, S.; Nolen, T.L.; et al. OnabotulinumtoxinA vs Sacral Neuromodulation on Refractory Urgency Urinary Incontinence in Women: A Randomized Clinical Trial. JAMA 2016, 316, 1366-1374. [CrossRef] [PubMed]

24. Amundsen, C.L.; Komesu, Y.M.; Chermansky, C.; Gregory, W.T.; Myers, D.L.; Honeycutt, E.F.; Vasavada, S.P.; Nguyen, J.N.; Wilson, T.S.; Harvie, H.S.; et al. Two-year outcomes of sacral neuromodulation versus OnabotulinumtoxinA for refractory urgency urinary incontinence: A randomized trial. Eur. Urol. 2018, 74, 66-73. [CrossRef] [PubMed]

25. Arlandis, S.; Castro, D.; Errando, C.; Fernandez, E.; Jimenez, M.; Gonzalez, P.; Crespo, C.; Staeuble, F.; Rodriguez, J.M.; Brosa, M. Cost-effectiveness of sacral neuromodulation compared to botulinum neurotoxin a or continued medical management in refractory overactive bladder. Value Health 2011, 14, 219-228. [CrossRef]

26. Martinson, M.; MacDiarmid, S.; Black, E. Cost of neuromodulation therapies for overactive bladder: Percutaneous tibial nerve stimulation versus sacral nerve stimulation. J. Urol. 2013, 189, 210-216. [CrossRef] [PubMed] 
27. Siddiqui, N.Y.; Amundsen, C.L.; Visco, A.G.; Myers, E.R.; Wu, J.M. Cost-effectiveness of sacral neuromodulation versus intravesical botulinum A toxin for treatment of refractory urge incontinence. J. Urol. 2009, 182, 2799-2804. [CrossRef] [PubMed]

28. Leong, R.K.; de Wachter, S.G.; Joore, M.A.; van Kerrebroeck, P.E. Cost-effectiveness analysis of sacral neuromodulation and botulinum toxin a treatment for patients with idiopathic overactive bladder. BJU Int. 2011, 108, 558-564. [CrossRef]

29. Wagg, A.; Franks, B.; Ramos, B.; Berner, T. Persistence and adherence with the new beta-3 receptor agonist, mirabegron, versus antimuscarinics in overactive bladder: Early experience in Canada. Can. Urol. Assoc. J. 2015, 9, 343-350. [CrossRef]

30. Yeaw, J.; Benner, J.S.; Walt, J.G.; Sian, S.; Smith, D.B. Comparing adherence and persistence across 6 chronic medication classes. J. Manag. Care Pharm. 2009, 15, 728-740. [CrossRef]

31. Carlson, K.; Civitarese, A.; Baverstock, R. OnabotulinumtoxinA for the treatment of idiopathic overactive bladder is effective and safe for repeated use. Can. Urol. Assoc. J. 2017, 11, e179-e183. [CrossRef] [PubMed]

32. Hoag, N.; Plagakis, S.; Pillay, S.; Edwards, A.W.; Gani, J. Sacral neuromodulation for refractory overactive bladder after prior intravesical onabotulinumtoxinA treatment. Neurourol. Urodyn. 2017, 36, 1377-1381. [CrossRef] [PubMed]

33. Martins-Silva, C.; Cruz, F. Efficacy and safety of OnabotulinumtoxinA in patients with urinary incontinence due to neurogenic detrusor overactivity: Update of the pivotal randomised, double-blind, placebo-controlled trials. Eur. Urol. Focus 2016, 2, 329-331. [CrossRef]

34. Banakhar, M.A.; Al-Shaiji, T.; Hassouna, M. Sacral neuromodulation and refractory overactive bladder: An emerging tool for an old problem. Ther. Adv. Urol. 2012, 4, 179-185. [CrossRef] [PubMed]

35. Siegel, S.W.; Catanzaro, F.; Dijkema, H.E.; Elhilali, M.M.; Fowler, C.J.; Gajewski, J.B.; Hassouna, M.M.; Janknegt, R.A.; Jonas, U.; van Kerrebroeck, P.E.; et al. Long-term results of a multicenter study on sacral nerve stimulation for treatment of urinary urge incontinence, urgency-frequency, and retention. Urology 2000, 56, 87-91. [CrossRef]

36. S Sterne, J.A.C.; Savovic, J.; Page, M.J.; Elbers, R.G.; Blencowe, N.S.; Boutron, I.; Cates, C.J.; Cheng, H.Y.; Corbett, M.S.; Eldridge, S.M.; et al. RoB 2: A revised tool for assessing risk of bias in randomised trials. BMJ 2019, 366, 14898. [CrossRef] 